\title{
Observations on the turkey oviductal sperm-storage tubule using differential interference contrast microscopy
}

\author{
M. R. Bakst \\ US Department of Agriculture, Agricultural Research Service, Avian Physiology Laboratory, \\ Livestock and Poultry Sciences Institute, Beltsville, MD 20705, USA
}

\begin{abstract}
Summary. Squash preparations of unfixed, uterovaginal junction mucosae revealed that openings to sperm-storage tubules were round or slit-like and were surrounded by either cilia, which were part of the uterovaginal junction surface epithelium, or nonciliated cells resembling the sperm-storage tubule epithelium. By focusing on different levels of the sperm-storage tubule (optical sectioning), connective tissue fibres and cells between individual sperm-storage tubules, epithelium and lumen of sperm-storage tubules containing resident spermatozoa were observed. An optical section through the sperm-storage tubule epithelium revealed basal nuclei and associated nucleoli, and refractile supranuclear lipid droplets. Luminal spermatozoa were distributed primarily in the distal third of the sperm-storage tubule and nearly always formed a tight bundle at its base. These spermatozoa were often observed slowly and synchronously oscillating. In two-thirds of the 30-week-old, non-photostimulated hens, sperm-storage tubules were fully formed. In contrast, the remaining hens possessed bud-like surface invaginations lacking discernible lumina. It was concluded that differential interference contrast microscopy offers better spatial and optical resolution of the sperm-storage tubule than other modes of light microscopy.
\end{abstract}

Keywords: sperm-storage tubules; oviduct; turkey

\section{Introduction}

The primary site of sperm storage in the bird oviduct is the sperm-storage tubules. In domestic poultry these tubules are collectively aggregated at the anterior end of the vaginal segment in a region referred to as the uterovaginal junction. The sperm-storage tubule has been the subject of several studies and reviews (Zavaleta \& Ogasawara, 1987; Bakst, 1987; Brillard, 1990). The aim of these studies was to relate the structure of the sperm-storage tubule to its capacity to store spermatozoa for prolonged periods after copulation or artificial insemination. Although light and electron microscopy have provided information on the histology and fine structure of the sperm-storage tubule and uterovaginal junction epithelia, such investigations have not revealed the physiological basis of sperm survival within the tubule.

In the course of our studies on oviductal sperm storage, it became necessary to develop or use techniques that present a better spatial appreciation of the sperm-storage tubule than that afforded through either histological sections, thin sections, or squash preparations viewed by phase contrast microscopy. The following study describes the use of differential interference contrast microscopy for examining squash preparations of fresh, unfixed uterovaginal junction mucosae containing sperm-storage tubules. New observations on the morphology of the sperm-storage tubules and the distribution of spermatozoa in the lumen of sperm-storage tubules are presented. 


\section{Materials and Methods}

\section{Animals and artificial insemination}

Turkey breeder hens and males were maintained under standard husbandry conditions in an environmentally controlled house. Feed and water were provided ad libitum. At 30 weeks of age, birds were photostimulated ( $14 \mathrm{~h}$ light: $10 \mathrm{~h}$ dark), and egg and semen production commenced 2-3 weeks later. Semen was manually collected from a minimum of 15 males, pooled and diluted $1: 1$ with a commercial turkey semen diluent before insemination. Hens were inseminated with $150 \times 10^{6}$ spermatozoa once a week during the 20 weeks of egg production.

\section{Preparation of sperm-storage tubules for microscopy}

Hens were killed with an overdose of sodium pentobarbital. The uterus and vagina were removed and handled as one segment. Fine forceps and scissors were used to gradually remove the connective tissue that held the vaginal segment in an ' $\mathrm{S}$ '-like configuration until the vaginal segment could be straightened. A longitudinal incision was then made through the vaginal and uterine segments and their mucosal folds revealed. With a rounded scalpel, the mucosa was gently scraped free of the underlying muscular layer. This sheet of mucosa was placed on a Petri dish and the mucosal folds were gently spread. Hank's Balanced Salt Solution (HBSS) was used to keep the mucosa moist. The mucosal spread was then viewed with a stereomicroscope to localize the region containing sperm-storage tubules. These procedures have been described, in part, by Bakst (1987) and Brillard \& Bakst (1990).

The sperm-storage tubules were prepared for a squash preparation by placing a piece of mucosa containing spermstorage tubules $(2 \mathrm{~mm} \times 2 \mathrm{~mm})$ with epithelial surface down on a clean slide. After adding $10 \mu$ of HBSS to the mucosa, any blood vessels or connective tissue were gently teased away from the mucosa. For the squash preparation, a clean $22 \mathrm{~mm} \times 30 \mathrm{~mm}$ coverglass (No. $I \frac{1}{2}$ ) was gently placed over the mucosa. If the specimen was small enough and the epithelial layer was not folded, the weight of the coverglass rendered the mucosa thin enough to be visualized by differential interference contrast microscopy. All procedures were performed at ambient temperature and no attempt was made to oxygenate the tissues. Slide specimens were examined with a Zeiss Universal Microscope with a Zeiss MC 63 camera attachment.

\section{Results}

Spermatozoa residing within individual sperm-storage tubules were found throughout the length of the tubules but were clearly more concentrated in the distal third (Figs 1 and 2). At the distal tip of the sperm-storage tubule, spermatozoa were aligned with their heads parallel and oriented toward the distal end of the tubule. This alignment was observed elsewhere only when both the spermstorage tubule made an acute turn and it was densely packed with spermatozoa (Fig. 3). Occasionally, spermatozoa would be slowly synchronously oscillating. Oscillations and other random motion of single spermatozoa were limited to the distal third of the sperm-storage tubule.

The surface epithelium lining the lumen of the uterovaginal junction was predominantly composed of ciliated cells. When viewed by differential interference contrast microscopy, only patches of contiguous cells were beating in synchrony, whereas cilia on adjacent cells remained immotile. Surface openings of individual sperm-storage tubules varied in appearance (Figs 4, 5). Some were round and nearly masked by the surrounding cilia. The beat of these cilia, which often extended a short distance into the sperm-storage tubule, created a circular beat pattern around the orifice of the tubule. (Using both enzymatic and mechanical means we have isolated separate intact spermstorage tubules, some still retaining the ciliated cells lining their orifice. The coordinated beat of these cilia created a vortex that would draw cell debris toward the orifice but not inside the spermstorage tubule.) The openings of other sperm-storage tubules appeared at the end of grooves or furrows formed by the surface mucosa of the uterovaginal junction. Here the slit-like openings of the tubules were either surrounded by a ciliated epithelium or a nonciliated epithelium characterized by the presence of large refractile granules, characteristically found in the epithelium of the sperm-storage tubules (Fig. 5). By observing a progression of optical sections, these slit-like openings led to a sperm-storage tubule which, after a short distance, bifurcated and formed two separate tubules (Fig. 6). Contrary to earlier reports (Bakst, 1987), additional branching of sperm-storage tubules, while infrequent, was also observed elsewhere along the length of the tubule. 


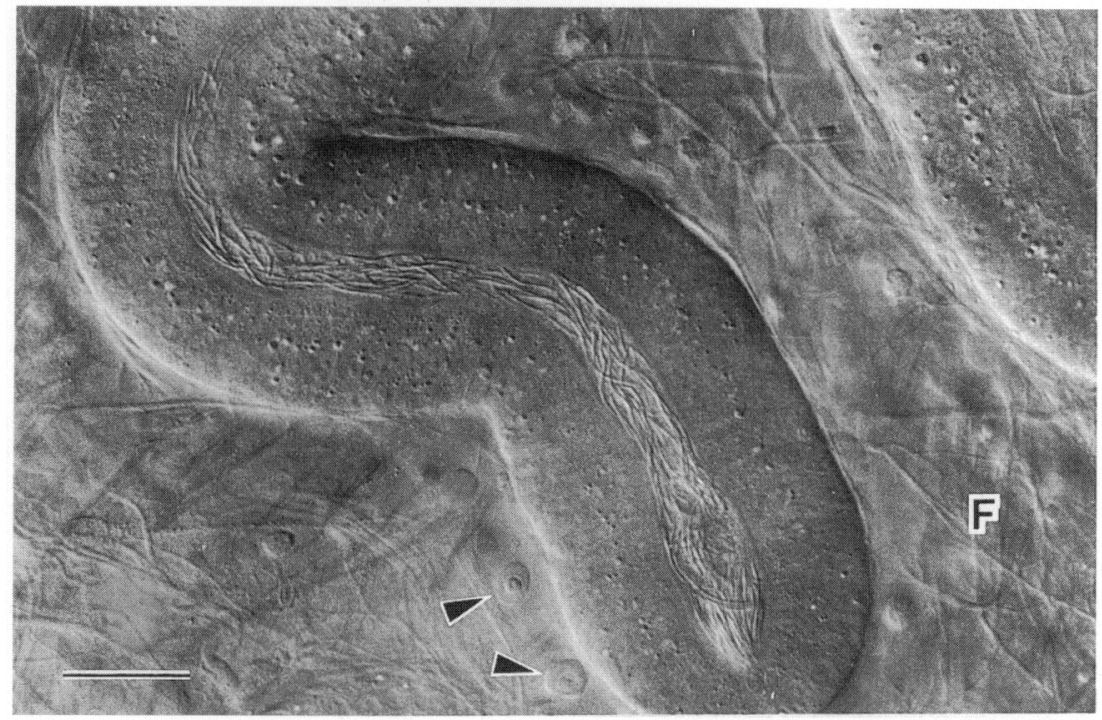

Fig. 1. Basal third of a single turkey sperm-storage tubule containing spermatozoa. Connective tissue fibres, a fibroblast (F) and extravascular erythrocytes (arrowheads) are present in the loose connective tissue. (Bar $20 \mu \mathrm{m}$.)

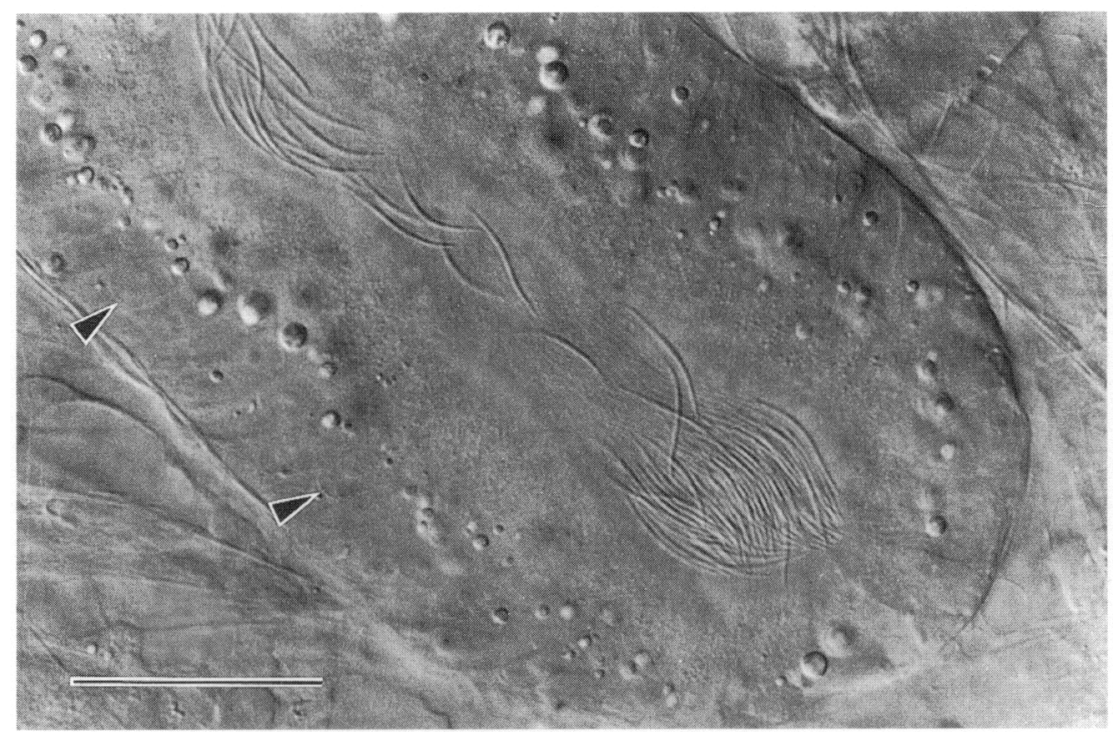

Fig. 2. Spermatozoa at the base of sperm-storage tubules typically form tight bundles with their heads aligned parallel and oriented towards the base of the tubule. Individual epithelial cells forming the tubule are evident as are cell nuclei (arrowheads). nucleoli and refractile supranuclear lipid droplets. (Bar $20 \mu \mathrm{m}$.)

At the basal aspect of the epithelium of the sperm-storage tubule, connective tissue fibres were closely apposed to the basement membrane of the tubule but did not appear to envelop the tubules (Figs 1, 2, 3). Only rarely were fibroblasts and other types of cell observed in the immediate vicinity of the tubule. However, in the surrounding loose connective tissue, small lymphocytes, heterophils, macrophages and blood vessels containing erythrocytes and granulocytes were 


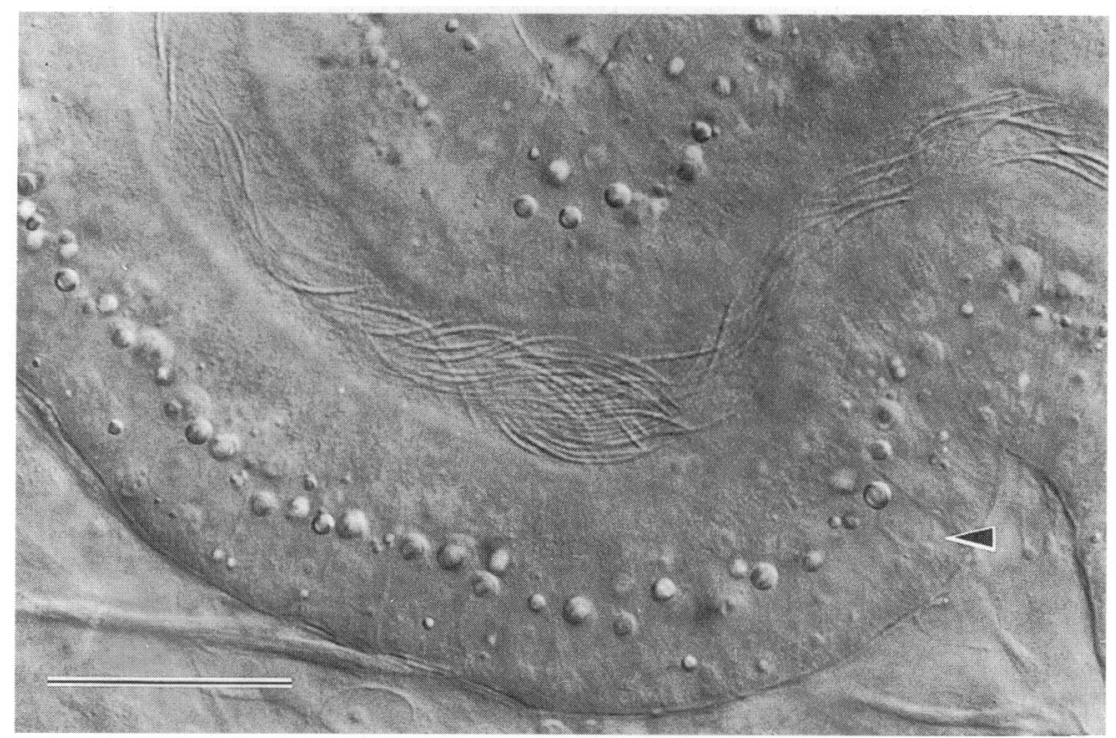

Fig. 3. Midportion of a sperm-storage tubule containing a secondary bundle of spermatozoa and other less densely distributed spermatozoa in the lumen. Individual epithelial cells of sperm-storage tubule and their nuclei (arrowhead), nucleoli and supranuclear lipid droplets are visible. ( $\operatorname{Bar} 20 \mu \mathrm{m}$.)

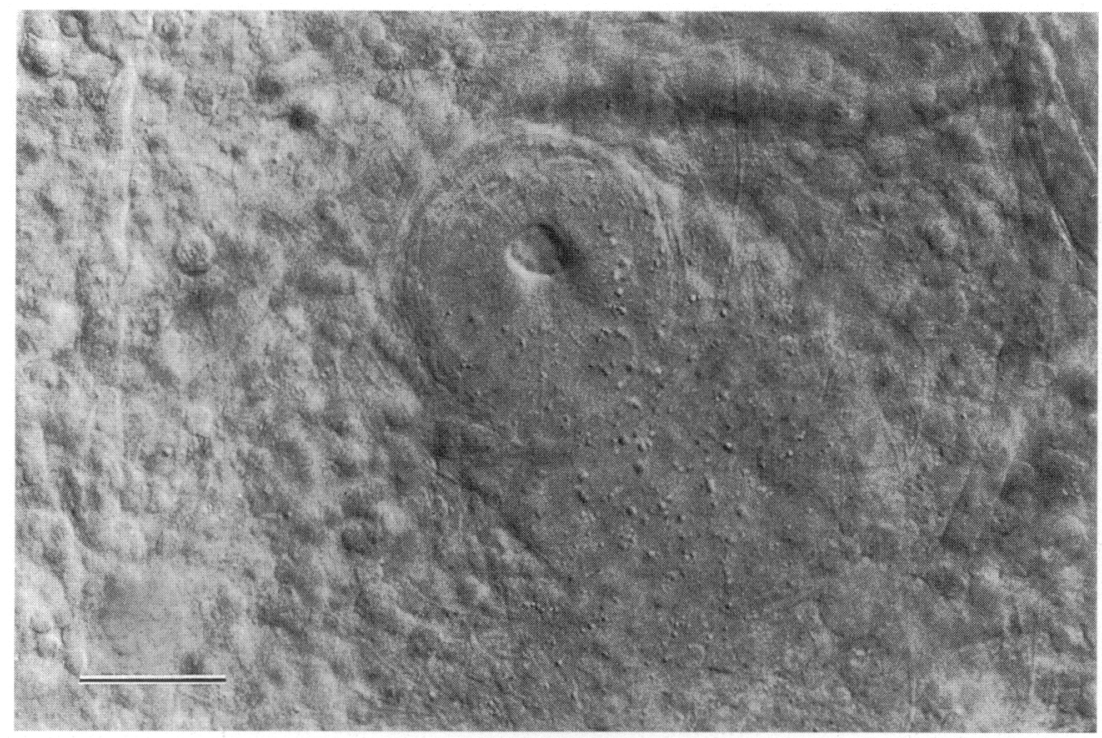

Fig. 4. The round orifice of a sperm-storage tubule is observed in an optical section at the level of the basal portion of the epithelial layer of the uterovaginal junction. (Bar $20 \mu \mathrm{m}$.)

readily apparent. By bringing the epithelium of the sperm-storage tubule into focus, individual simple columnar epithelial cells characterized by a basal nucleus with a prominent nucleolus were observed (Figs 2, 3). Highly refractile lipid droplets were found in the supranuclear cytoplasm (Figs 2, 3). Their size and number within sperm-storage tubules both within and between hens varied but had no relationship to the presence or absence of spermatozoa within individual tubules. 


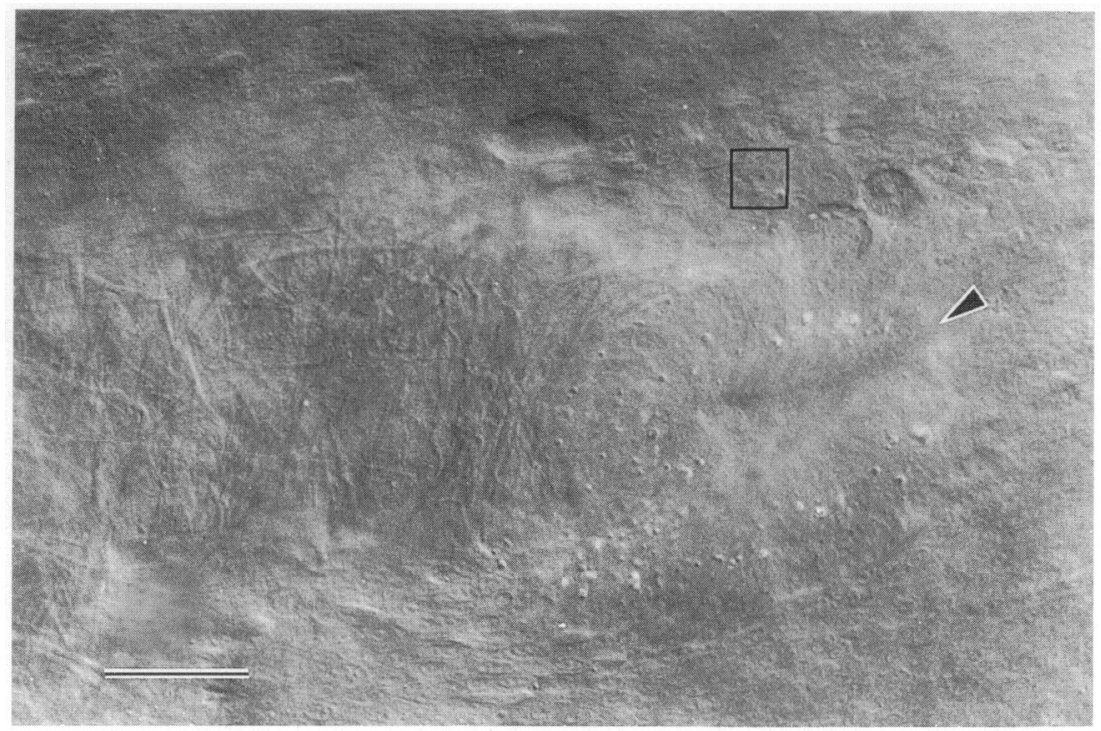

Fig. 5. A slit-like opening to a sperm-storage tubule (arrowhead) is observed enveloped by a raised area formed by an epithelium similar to that lining the tubule. Profiles of uterovaginal junction epithelial cell nuclei (within square) and connective tissue fibres surrounding the tubule are also discernible. (Bar $20 \mu \mathrm{m}$.)

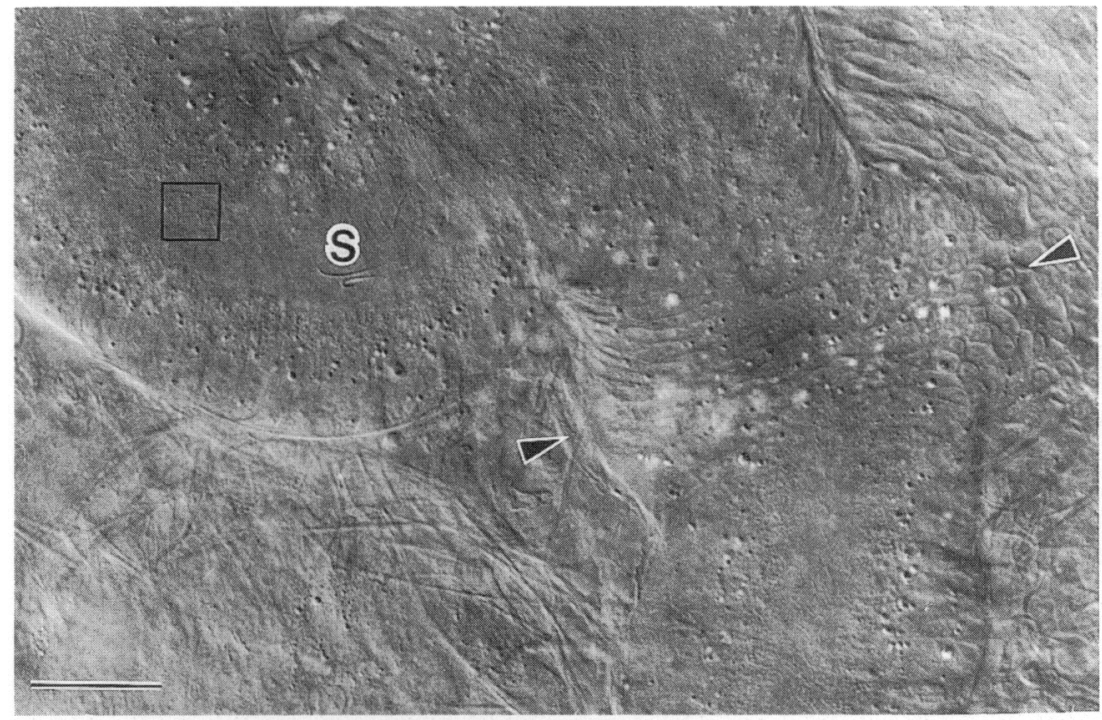

Fig. 6. Optical section of the subepithelial region of uterovaginal junction epithelium in turkey. A short distance from the surface opening a sperm-storage tubule bifurcates (arrowheads) and forms two tubules. In the lumen of one tubule are two spermatozoa (S) and margins of the sperm-storage tubule epithelial cells (within square). (Bar $20 \mu \mathrm{m}$.)

The granular appearance of the apical cytoplasm in the epithelium of sperm-storage tubules (Figs 2, 3, 7) was probably due to organelles such as lysosomes, mitochondria and aggregates of membrane-bound vesicles (Schuppin et al., 1984). When focusing on the luminal surface of the tubules, raised, polygonal-shaped cell borders, possibly a manifestation of tight junctions, were observed (Fig. 6). 


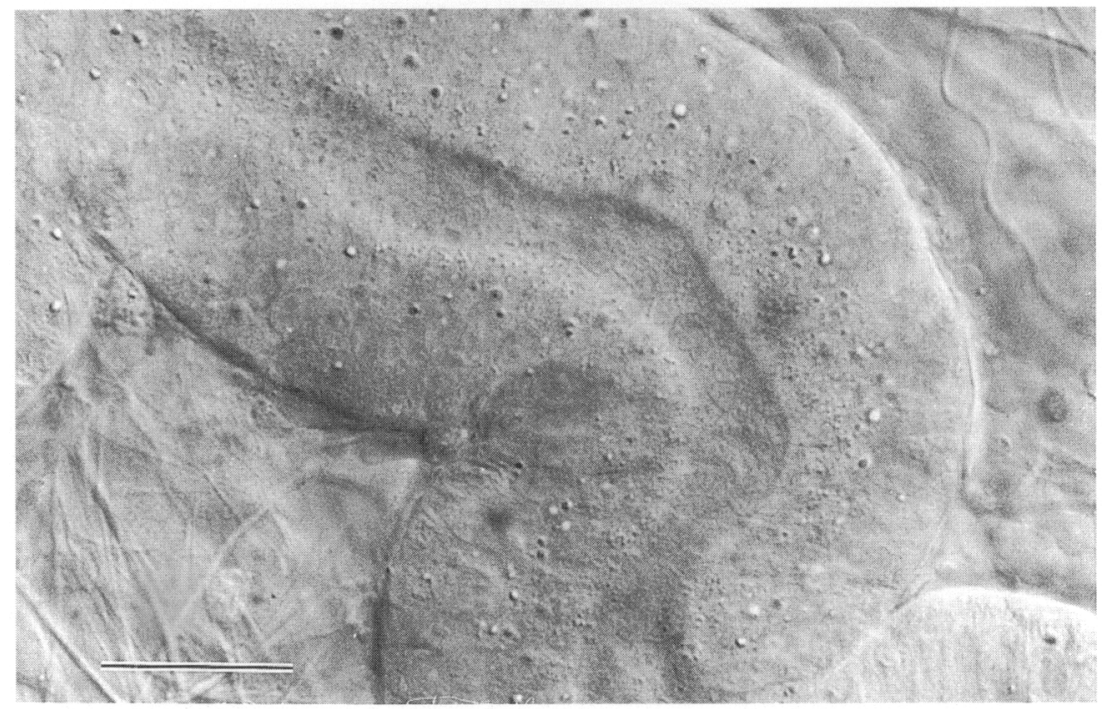

Fig. 7. A differentiated sperm-storage tubule from a 30-week-old hen at the onset of photostimulation showing similar morphology to those observed in hens already in egg production. (Bar $20 \mu \mathrm{m}$.)

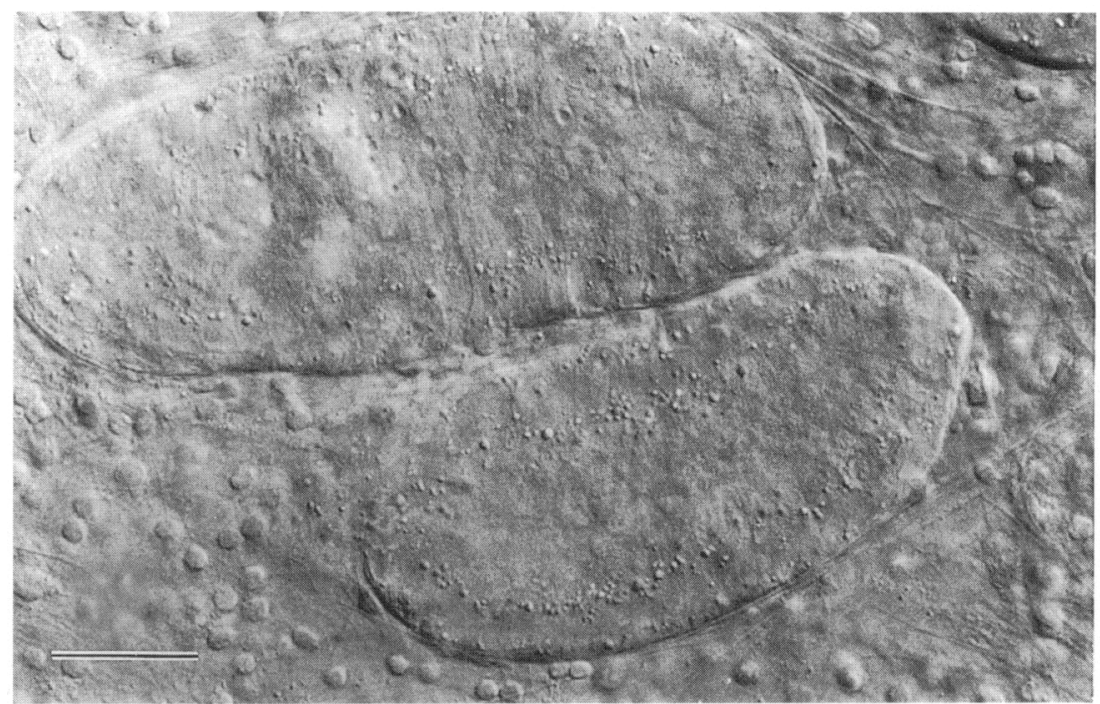

Fig. 8. Two bud-like sperm-storage tubules are observed within the uterovaginal junction mucosa of a 30-week-old hen. The cells in the loose connective tissue compartment are probably small lymphocytes. (Bar $20 \mu \mathrm{m}$.)

The differential interference contrast microscopy procedure has been used to evaluate the development of sperm-storage tubules in hens both at the onset of photostimulation at 30 weeks of age and earlier. Of the 12 hens examined at the onset of photostimulation, eight had fully formed sperm-storage tubules (Fig. 7). The remaining had bud-like structures with no discernible lumina (Fig. 8). Transition forms of sperm-storage tubules were not observed. 


\section{Discussion}

When squash preparations of the mucosae of the uterovaginal junction were observed by differential interference contrast microscopy, individual intact sperm-storage tubules were clearly visible. Unlike observing the same type of preparation by phase contrast microscopy (Bakst, 1987), differential interference contrast microscopy allowed clear visualization of the features of the tubular epithelium, the spatial relationship of the sperm-storage tubules and the surrounding connective tissue and, for the first time, the capacity to view the distribution of spermatozoa throughout the length of the tubule. Similar observations were possible previously only with the reconstruction of serial sections of fixed and embedded tissues. Differential interference contrast microscopy is compatible with fluorescence microscopy and we are currently using differential interference contrast microscopy and fluorescence microscopy to elucidate mechanisms of selection and transport of spermatozoa in the sperm-storage tubules and vagina.

With regard to the disposition of spermatozoa within the sperm-storage tubule, most spermatozoa within the turkey sperm-storage tubules are not arranged in organized, head-to-head agglutinated bundles, as described for chickens by Van Krey et al. (1981). These authors suggested that the capacity of resident spermatozoa to agglutinate within the sperm-storage tubules was the underlying mechanism controlling sperm storage within the sperm-storage tubule of the chicken. Observations in this study indicate that head-to-head agglutination is not a prerequisite for successful sperm storage in the turkey.

The presence of fully differentiated and functional sperm-storage tubules in two-thirds of the 30-week-old hens examined before the onset of photostimulation would support the previous contention that oestrogen and progesterone originating from maturing yellow-yolk follicles are not necessarily the main effectors of sperm-storage tubule growth and differentiation (Bakst, 1988). However, with the onset of photostimulation and the extremely rapid growth and maturation of the ovary and oviduct, increased circulating levels of oestrogen and progesterone would probably function in the differentiation of new sperm-storage tubules (Pescatore \& Marquez, 1977) and the elongation and lumen formation of pre-existing bud-like sperm-storage tubules. The latter event must be rapid since a transition between sperm-storage tubules having no discernible lumina and fully formed sperm-storage tubules have not been observed.

\section{References}

Bakst, M.R. (1987) Anatomical basis of sperm-storage in the avian oviduct. Scanning Microscopy 1, 1257-1266.

Bakst, M.R. (1988) Duration of fertility of turkeys inseminated at different times after the onset of photostimulation. Journal of Reproduction and Ferility 84, $531-537$.

Brillard, J.P. (1990) Stockage des spermatozoides dans l'oviducte chez les oiseaux: approche morphologique, histologique et fonctionnelle. Reproduction Nutrition Développement 30, 16I-174.

Brillard, J.P. \& Bakst, M.R. (1990) Quantification of spermatozoa in the sperm-storage tubules of turkey hens and the relation to sperm numbers in the perivitelline layer of eggs. Biology of Reproduction 43, 271-275.

Pescatore, A.J. \& Marquez, B.J. (1977) Sperm storage tubule developmet in immature turkeys injected with female sex steroids. Poultry Science 56, 1746-1747.
Schuppin, G.T., Van Krey, H.P., Denbow, D.M., Bakst, M.R. \& Meyer, G.B. (1984) Ultrastructural analyses of uterovaginal sperm storage glands in fertile and infertile turkey breeder hens. Poultry Science 63, 18721882 .

Van Krey, H.P., Balander, R.J. \& Compton, M.M. (1981) Storage and evacuation of spermatozoa from the uterovaginal sperm-host glands in domestic fowl. Poultry Science 60, 871-877.

Zavaleta, D. \& Ogasawara, F. (1987) A review of the mechanism of the release of spermatozoa from storage tubules in the fowl and turkey oviduct. World's Poultry Science Journal 43, 132-139.

Received 9 July 1991 